\title{
Role of proton Pump Inhibitors in the Management of Laryngopharyngeal Reflux
}

\author{
Authors \\ Vellavedu Umapathy Shanmugam, Gurusamy Satheeshkumar, \\ Ruta Shanmugam, Balaji Swaminathan, Premnivas \\ Department of Otorhinolaryngology, Rajah Muthiah Medical College, Chidambaram
}

\begin{abstract}
Back Ground: The study was conducted to observe different signs and symptoms of Laryngopharyngeal reflux and its management and outcome with proton pump inhibitor therapy.

Methods: This prospective Study was conducted at Rajah Muthiah Medical College Hospital in the department of Otorhinolaryngology, from October 2018 to September 2019, among 30 Patients who presented to the hospital with symptoms and signs of Laryngopharyngeal reflux disease. The different signs and symptoms of Laryngopharyngeal reflux and the role of proton pump inhibitors in the management using Reflux Finding Score (RFS) and Reflux Symptom Index (RSI) were studied.

Results: The mean age group was 43.5 years. Foreign body sensation in the throat and frequent clearing of throat were most common presenting symptoms and Erythema of the arytenoids along with posterior commisure hypertrophy and ventricular obliteration were the common finding in videolaryngoscopy in patients of Laryngopharyngeal reflux. There was a significant improvement in the reflux symptom index and reflux finding score following treatment with proton pump inhibitors.

Conclusion: Proton pump inhibitors is an effective treatment modality in the management of laryngopharyngeal reflux. The reflux finding score and reflux symptom index of Wake Forest University are valuable tools for diagnosing LPR.
\end{abstract}

Keywords: Laryngopharyngeal reflux, GERD, Proton pump inhibitors.

\section{Introduction}

The term Laryngopharyngeal Reflux (LPR) was coined by James and is accepted by the American Academy of Otolaryngology: Head and Neck surgery. ${ }^{[1]}$ Laryngopharyngeal reflux disease (LPRD) was first described by von Leden and Moore, in the 1960, but it did not come to the forefront of otolaryngology practice until Koufman's landmark thesis on the subject in $1991{ }^{[1,2]}$ Laryngopharyngeal reflux disease is an extraesophageal variant of gastroesophageal reflux disease that affects the larynx and pharynx. ${ }^{[3,4]}$ The other terms used for this in otorhinolaryngology practice are 'extra esophageal reflux', 'chronic laryngitis' and 'supra esophageal complication of gastroesophageal reflux'. ${ }^{\text {[4] }}$ Recent studies in this field evidently proves that laryngopharyngeal reflux represents a complex spectrum of abnormalities and it is therefore important to understand the basic scientific concepts relevant to this disease and also the appropriate clinical care of patients with laryngopharyngeal reflux.

The incidence of patients presenting to an 
Otolaryngologist with GERD has been estimated to be $4 \%$ to $10 \% .{ }^{[10]}$ Though Laryngopharyngeal reflux disorder is a common scenario in clinical practice, it is usually under reported, misdiagnosed or neglected for want of a definitive diagnosis. The prevalence of laryngopharyngeal reflux disease is very high. ${ }^{[7,8]}$ There are no epidemiological studies to show us the prevalence of laryngopharyngeal reflux disease in India. The aim of the study is to evaluate the role of Proton pump inhibitors in the management of Laryngopharyngeal reflux disease.

\section{Materials and Methods}

The Study was conducted in the department of otorhinolaryngology, at Rajah Muthiah Medical College Hospital for duration of one year between October 2018 to September 2019. Totally 30 patients with symptoms and signs of LPRD (RSI $>13$, RFS > 7) were included in the study. Patients with RFS less than 7 and/or RSI less than 13, Patients with some other obvious cause of symptoms and signs like infection, malignancy, history of antireflux medication in the preceding one month were excluded from the study.

After obtaining ethical committee approval, informed and signed consents were obtained from all the patients under the study. Complete history taking, and thorough ENT examination was done to exclude other causes, Videolaryngoscopy was done for all patients. Proton Pump Inhibitors like Omeprazole $20 \mathrm{mg}$ twice daily or Pantoprazole 40 $\mathrm{mg}$ twice daily was used in the study.Patients were followed for sixteen weeks, on two occasions first at 8 weeks then at 16 weeks. On each follow up visit patients symptoms were evaluated with RSI and laryngoscopic findings scored with RFS. Effect of Proton Pump Inhibitors on RSI and RFS at each follow up visit was used to assess the role of PPI. The results were tabulated and statistically analysed using SPSS version 17.0.

\section{Results}

In our study majority of cases were in the age groups of 41-50 years. The average mean was 43.5 years, the youngest patient was 19 years old and the oldest patient was 72 years old.

Graph: 1 Age distribution

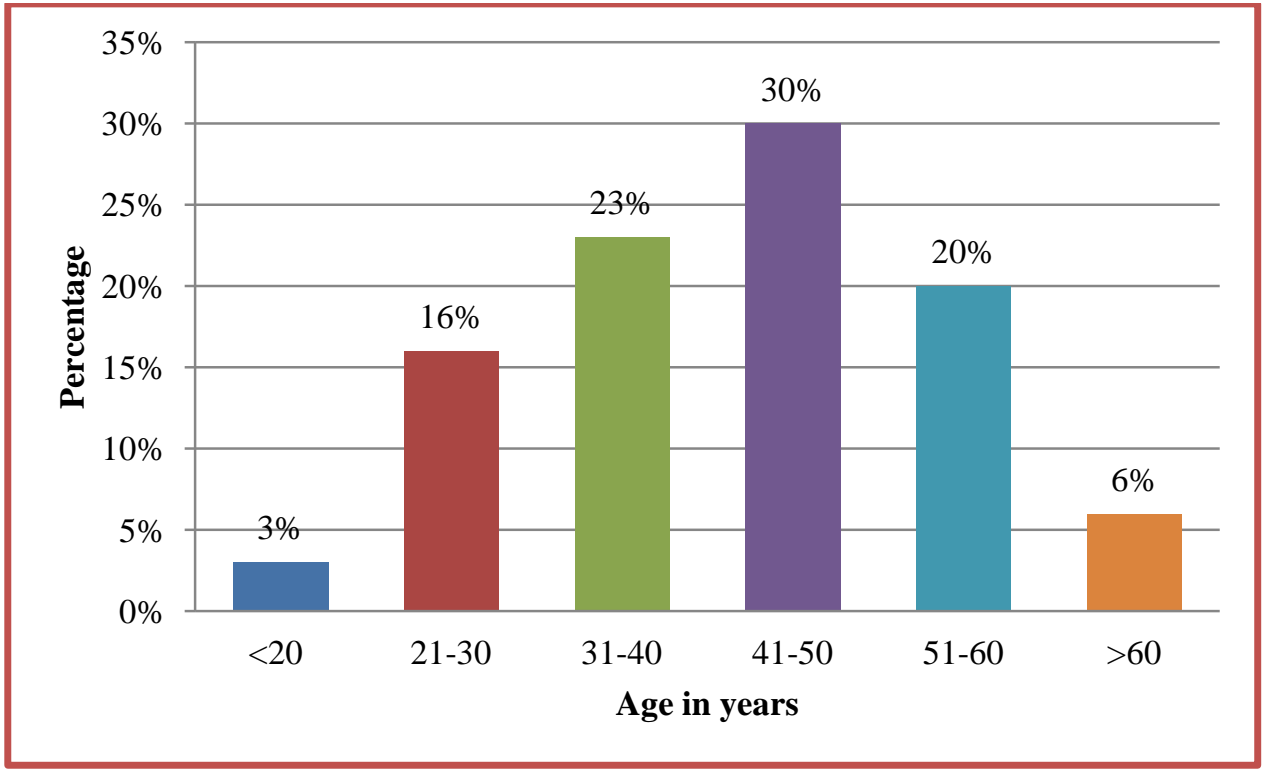

\section{Gender Distribution}

Out of 30 patients, 12 were male and 18 were females. Majority of patients were females. 
Graph 2. Gender distribution

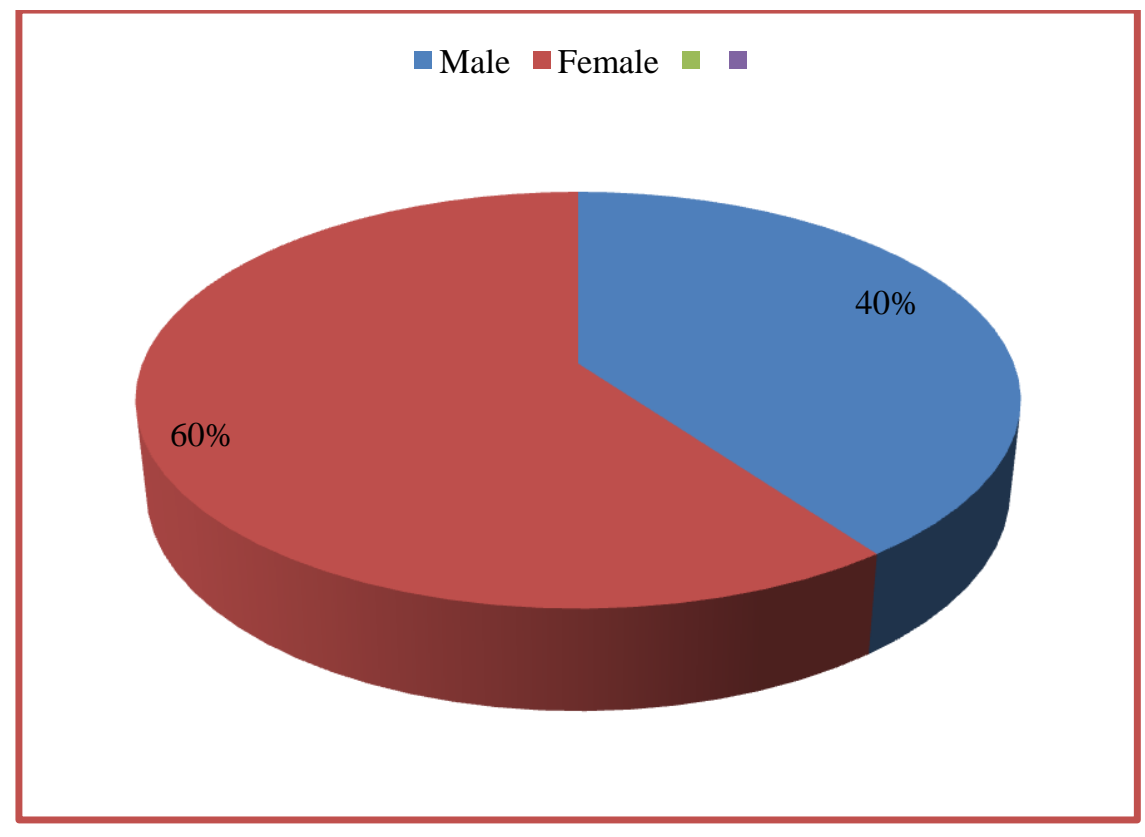

Mean RSI of all patients was 24.80 before treatment with PPIs. After 8 weeks of therapy with PPI mean RSI decreased to 14.53 and after 16 weeks of PPI therapy mean RSI dropped to
12.96.Significant change in RSI occurred after first 8 weeks of therapy in total in all age groups and further significant change occurred in the next 8 weeks.

Graph: 3 Percentage distribution of symptoms (RSI)

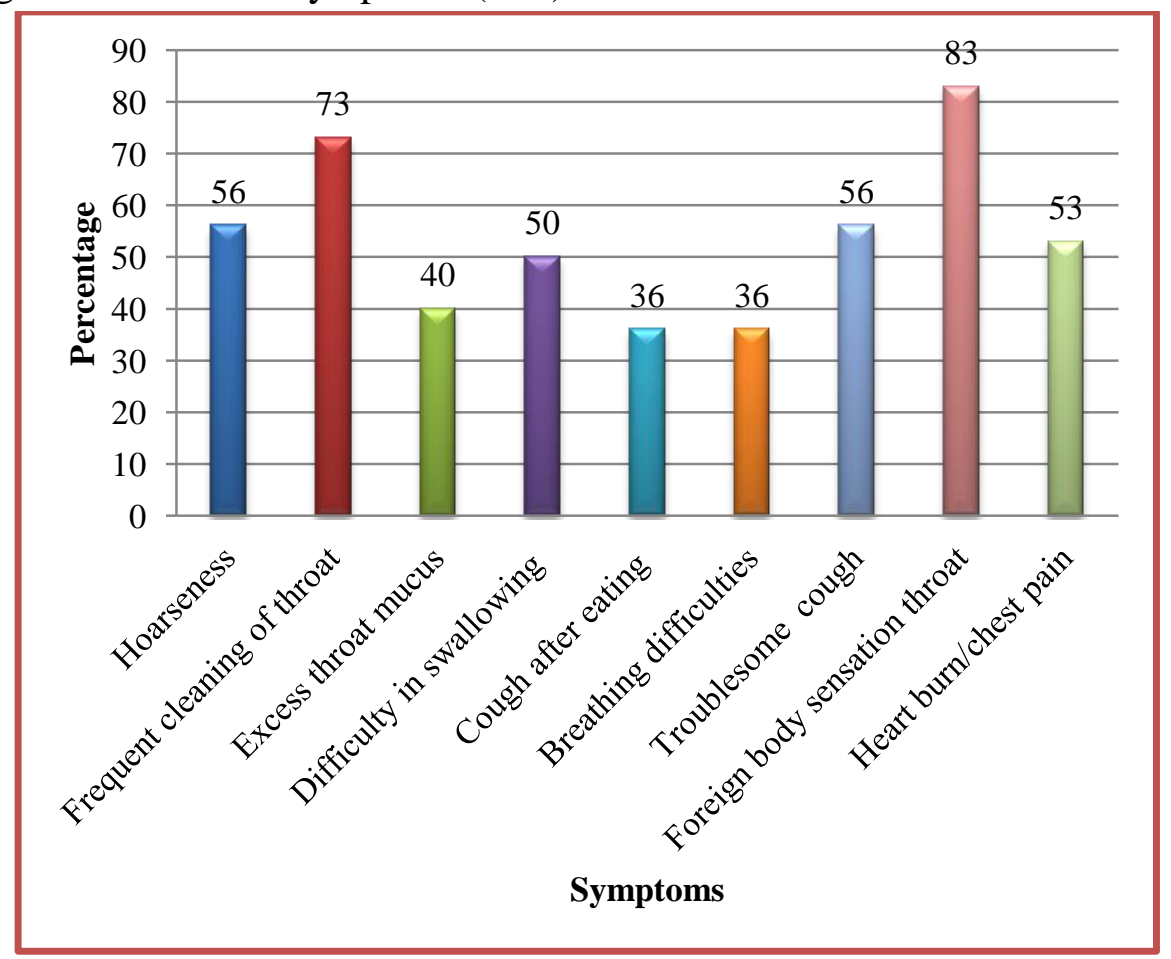

Foreign body sensation was the most common symptom present in $83 \%$ of patients next was frequent clearing of throat in $73 \%$, troublesome or annoying cough and hoarseness in $56 \%$ of patients. 
Graph: 4 RSI pre and post PPI therapy

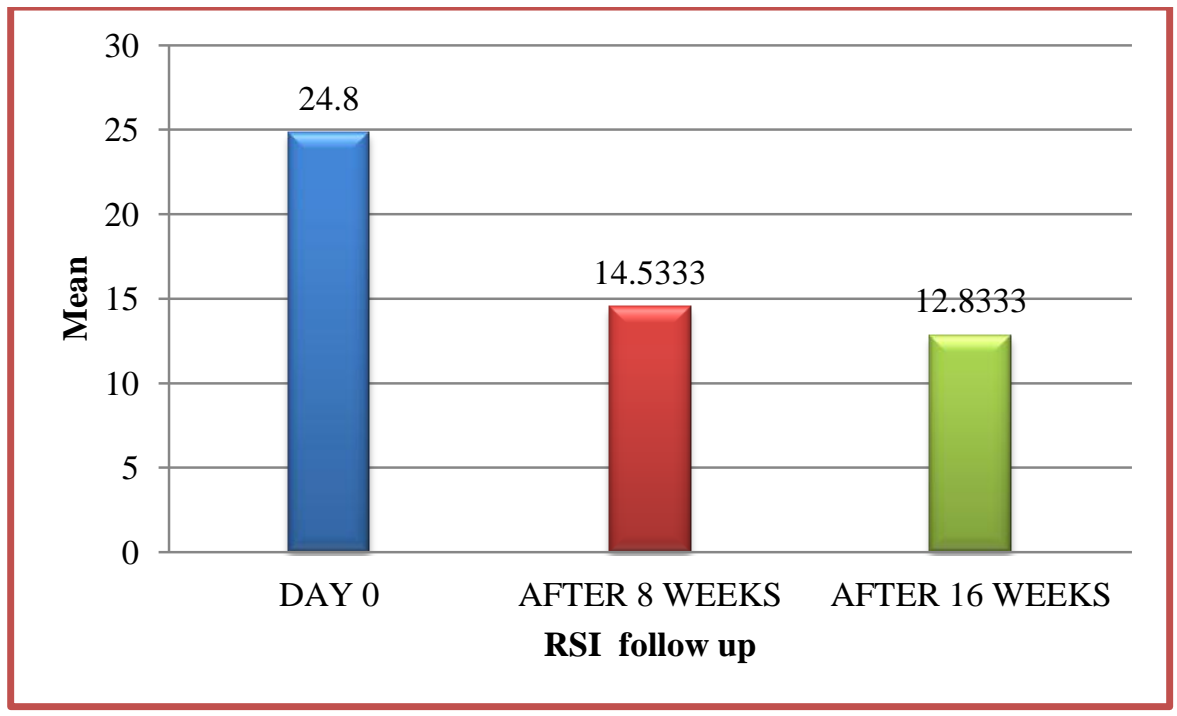

Mean RFS of the patients was 12.66 before treatment with proton pump inhibitors. After 8 weeks of therapy with PPI mean RFS decreased to
9.46 and after 16 weeks of PPI therapy mean RFS dropped to 6.80 .

Graph: 5 RFS findings

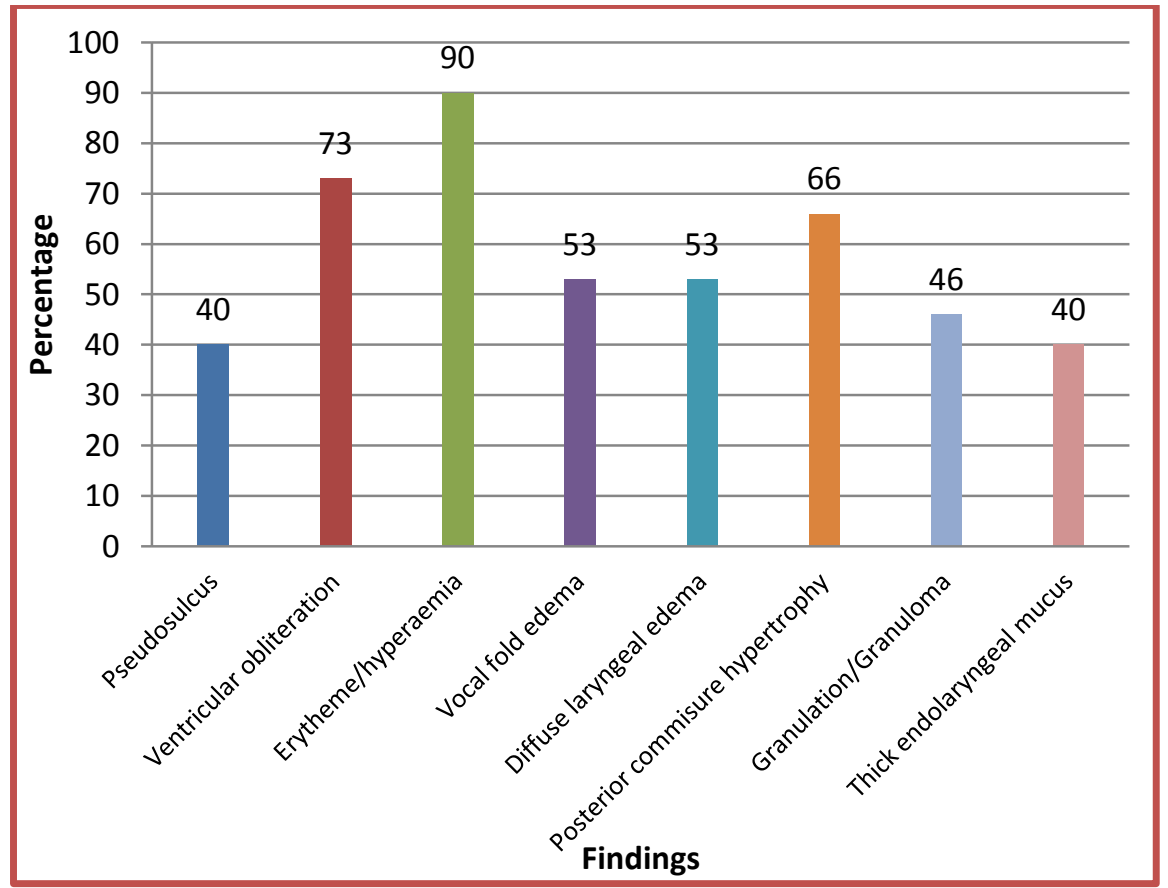

\section{Percentage distribution of symptoms (RSI)}

Most common laryngeal finding was erythema / hyperaemia $90 \%$, next was ventricular obliteration $73 \%$ and posterior commisure hypertrophy $66 \%$. 
Graph: 6 RFS pre and post PPI therapy

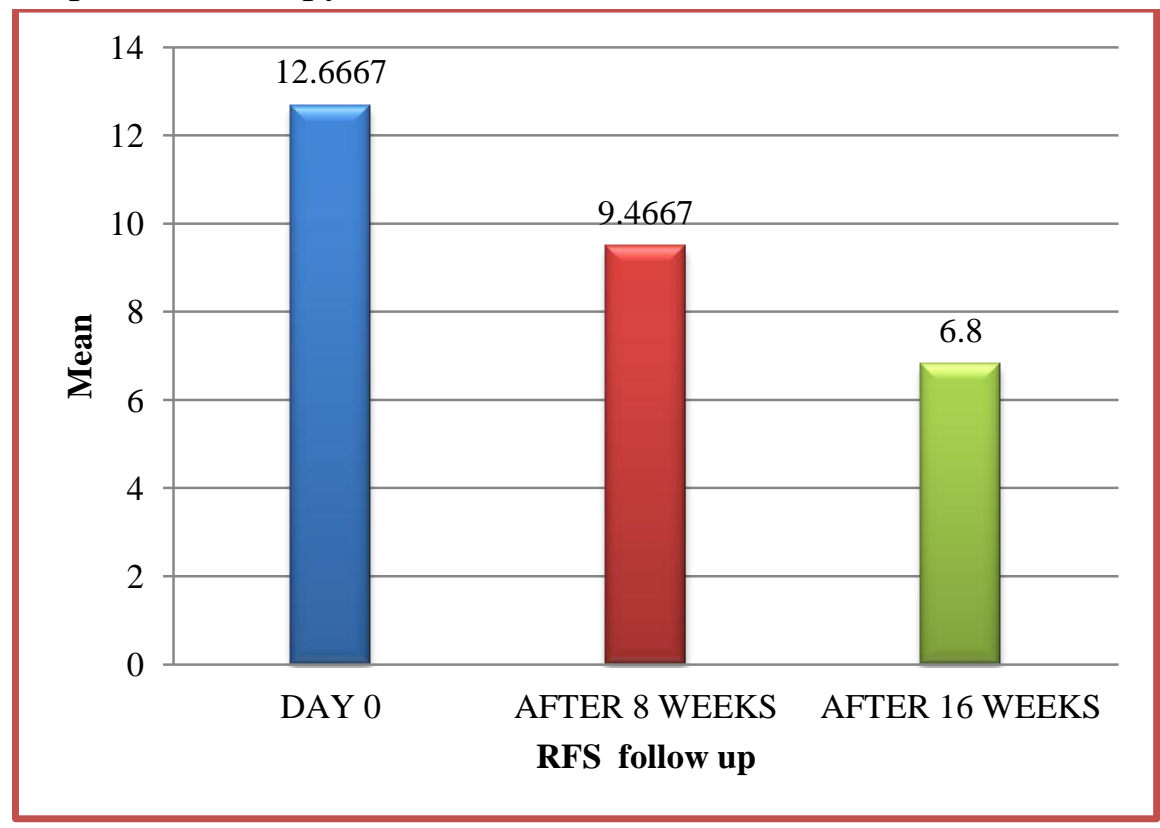

Mean RFS of the patients was 12.66 before treatment with PPIs. After 8 weeks of therapy with PPIs mean RFS decreased to 9.46 and after 16 weeks of PPIs therapy mean RFS dropped to 6.80 .

\section{Discussion}

The present study was aimed to evaluate the effect of Proton pump inhibitors on the reflux symptom index and reflux finding score among those who suffer from Laryngopharyngeal reflux disease. This study included 30 patients. Those patients with other co-existing laryngeal pathology, patients who are hyper sensitive to proton pump inhibitors, who are on proton pump inhibitor therapy for the past one month and pregnant women were excluded from the study. Out of 30 patients mean age was 43.5 years which was similar to study conducted by Matto $\mathrm{O}$ et al $(2012)^{[6]}$. Gender based distribution of the subjects in our study revealed that there was a female predominance $60 \%$ which was similar to study done by Patigaroo et al (2012) $60 \%{ }^{[9]}$. However study done by Belafsky et al.(2002) ${ }^{[8]}$ showed male predominance $56 \%$.In our study the mean reflux symptom index for pre-treatment group was 24.80 and it was reduced to 12 in the post treatment group. Wilcoxons signed rank test was done for the pre-treatment and post treatment group and it gave a $\mathrm{p}<0.000$ which is highly significant. Niran Hunchaisri et al in Bangkok , Thailand compared between Domperidone plus omeprazole and omeprazole alone. Total reflux symptom index were compared between groups before treatment. After three months of the treatment, moderate improvement of total RSI and individual sub scores were statistically significant with each group $(\mathrm{p}<0.001$ each $) .{ }^{[11]}$

Anuja Bhargava et al in lucknow, India in 2018 published a prospective study to evaluate Role of Proton pump inhibitors in Laryngopharyngeal reflux disease. This study was undertaken to evaluate the effect of various proton pump inhibitors in the treatment of Laryngopharyngeal reflux disease using reflux symptom index and reflux finding score. After 3 months, reflux symptom index score improved significantly with Proton pump inhibitor theray. ${ }^{[12]}$ Nasir A. Khan et al in 2014 published a prospective study in Laryngopharyngeal reflux disease patients. Frequent clearing of throat was the most common symptom. Mean reflux symptom index of all patients was 25.25 before treatment. Significant change in reflux symptom index occurred after first 10 weeks of therapy and no further significant change occurred in the next 10 weeks. ${ }^{[13]}$ In our study the comparison of reflux symptom index of pre-treatment group with that of the post 
treatment group showed a difference of 6 i.e. a reduction from 12.66 to 6.80 with 16 weeks of treatment. The data was analysed used Wilcoxons signed rank test and it showed a $\mathrm{p}$ value 0.000 which is significant.

Suhail A Patigaroo and colleagues' in their study of 50 Indian patients with Laryngopharyngeal reflux disease, showed a mean reduction in Reflux finding score from 12 in the pretreatment to 6.5 in the post treatment group ${ }^{[9]}$. These values are comparable to our study. Anuja Bhargava et al in lucknow, India in 2018 published a prospective study to evaluate Role of Proton pump inhibitors in Laryngopharyngeal reflux disease. After 3 months, RFS score improved significantly with Proton pump inhibitor theray. ${ }^{[12]}$ Nasir A .Khan et al in 2014 published a prospective study in Laryngopharyngeal reflux disease patients. Most common laryngeal finding was erythema / hyperaemia; Mean reflux finding score of all patients was 13 before treatment with Proton pump inhibitors. There was slight response after first 10 weeks of therapy in physical findings and significant response after 20 weeks of therapy. ${ }^{[13]}$ Our study didn't show any gender difference, in the reduction of reflux symptom index or reflux finding score.

\section{Conclusion}

The incidence of the laryngopharyngeal reflux is more predominant in females compared to males in the ratio 3:2.The mean age group in our study is 43.53 \pm 13 .33. Foreign body sensation in the throat and frequent clearing of throat were the most common presenting symptoms in patients with laryngopharyngeal reflux. Erythema of the arytenoids along with posterior commisure hypertrophy and ventricular obliteration were the common finding in videolaryngoscopy. There was a significant improvement in the reflux symptom index and reflux finding score following treatment with proton pump inhibitors. Although all our patients responded to treatment failures as reported in literature are not uncommon.

\section{References}

1. Koufman JA. The otolaryngologic manifestations of gastroesophageal reflux disease (GERD): a clinical investigation of 225 patients using ambulatory 24-hour $\mathrm{pH}$ monitoring and an experimental investigation of the role of acid and pepsin in the development of laryngeal injury. Laryngoscope 1991; $101(4 \quad \mathrm{Pt} 2$ Suppl53):1-78.

2. Sataloff RT, Hawkshaw MJ, Gupta R. Laryngopharyngeal reflux and voice disorders: an overview on disease mechanisms, treatments, and research advances. Discov Med. 2010 Sep;10(52):213-24.

3. Castell DO, Murray JA, Tutuian R, Orlando RC, Arnold R. Review article: the pathophysiology of gastro-oesophageal reflux disease - oesophageal manifestations. Aliment Pharmacol Ther2004;20Supp19:14-25.

4. Habermann W, Schmid C, Neumann K, DevaneyT, Hammer HF. Reflux symptom index and reflux finding score in otolaryngologic practice. J Voice.2012May;26(3):e123-7.

5. Kondo Y, Ogasawara N, Sasaki M, Arimoto M, Yanamoto K, Nishimura K et al. Edema of the interarytenoid mucosa seen on endoscopy is related to endoscopic-positive esophagitis (EE) and is an independent predictor of EE. Dig Endosc.2013;25(6):578-84.

6. Mattoo O, Muzaffar R, Mir A, YousufA, Charag AH, Ahmad R. Laryngopharyngeal Reflux: Prospective study analysing various non-surgical treatment modalities for LPR. Int $\mathrm{J}$ PhonosurgLaryngol.2012;2(1):5-8.

7. Koufman JA, Amin M, Panetti M. Prevalence of reflux in 113 consecutive patients with laryngeal and voice disorders. Otolaryngol Head Neck Surg.2000;123:385-8. 
8. Belafsky PC, Postma GN, KoufmanJA.

Validity and reliability of the reflux symptom index (RSI). J Voice.2002;16(2):274-7.

9. Patigaroo SA, Hashmi SF, Hasan SA, Ajmal MR, Mehfooz N. Clinical manifestations and role of proton pump inhibitors in the management of laryngopharyngeal reflux. Indian J Otolaryngol Head Neck Surg. 2011 Apr;63(2):182-9.

10. KoufmanJA, Aviv JE, Casiano RR et al. Laryngopharyngeal reflux: position statement of the committee on speech, voice, and swallowing disorders of the American academy of OtolaryngologyHead and Neck Surgery. Arch Otolaryngol Head Neck Surg. 2002; 127-32.

11. Niran Hunchaisri MD* Treatment of Laryngopharyngeal Reflux : A Comparison between Domperidone Plus Omeprazole and Omeprazole Alone . J Med Assoc Thai 2012; 95 (1): 73-80

12. Anuja Bhargava et al. Role of proton Pump Inhibitors in Laryngopharyngeal Reflux: Clinical Evaluation in a North Indian Population. Indian journal of Otolaryngology and Head \& Neck Surgery 2018.

13. Nazir A. Khan et al. Laryngopharyngeal Reflux and Proton Pump Inhibitors. Indian Medical Gazette - May 2014. 